\title{
Pain and motor complications in Parkinson's disease
}

\author{
M Tinazzi, C Del Vesco, E Fincati, S Ottaviani, N Smania, G Moretto, A Fiaschi, \\ D Martino, G Defazio
}

See end of article for authors' affiliations

.....................

Correspondence to: Dr M Tinazzi, Unità Operativa di Neurologia OC Borgo Trento, Piazzale Stefani 1, 37100 Verona, Italy; michele.tinazzi@ mail.azosp.vr.it

Received 24 August 2005 Revised version received 22 February 2006

Accepted 10 March 2006

Published Online First

20 March 2006

\begin{abstract}
Aims: To study the association of pain with motor complications in 117 patients with Parkinson's disease. Methods: Patients were asked to refer any pain they experienced at the time of study and lasting since at least 2 months. Basic parkinsonian signs and motor complications (including motor fluctuations and dyskinesia) were assessed and Unified Parkinson's Disease Rating Scale (UPDRS) motor score part III (during on) and part IV were calculated. Information on age, sex, duration of disease, use of dopamine agonists and levodopa, years of levodopa treatment and current levodopa dosage, medical conditions possibly associated with pain, and depression were collected. Single and multiple explanatory variable logistic regression models were used to check the association of pain with the investigated variables.

Results: Pain was described by 47 patients (40\%) and could be classified into dystonic (n.19) and non dystonic pain (n. 16); in 12 patients both types coexisted. Multiple explanatory variable logistic regression models indicated a significant association of pain with motor complications (adjusted OR, 5.7; 95\% Cl, 2 to $16.5 ; p=0.001$ ). No association was found between pain, dystonic or non dystonic, and the other investigated variables including medical conditions known to be associated to pain in the general population. There was a significant correlation $(r=0.31, p<0.05)$ between severity of pain (measured on a Visual Analogue Scale) and severity of motor complications (UPDRS part IV).

Conclusions: Pain may be a representative feature of Parkinson's disease frequently associated with motor complications. The association is independent of a number of potentially relevant demographic and clinical variables.
\end{abstract}

State Examination score <24) were excluded. A neurologist trained in movement disorders assessed basic parkinsonian signs (rest tremor, rigidity and bradykinesia) and motor complications (including motor fluctuations and dyskinesia). Unified Parkinson's Disease Rating Scale (UPDRS) motor scores part III (during the on state) and part IV were calculated. Information on age, sex, duration of disease, use of dopamine agonists and levodopa, years of levodopa treatment and current levodopa dosage were collected.

All patients were informed about the nature of the study and gave their consent for participation. The ethics committee of the University of Verona Hospital approved the study.

Patients were asked to refer to and describe any pain they had experienced at the time of study and that had lasted for at least 2 months. According to previous reports, the pain that was reported was classified in the following categories: musculoskeletal (aching, cramping, arthralgic, joint), radicular-neuropathic (pain in the territory of a root or nerve), dystonic (pain associated with dystonic movements and postures), central primary (burning, tingling, formication, bizarre quality) and akathitic discomfort. ${ }^{8}$ Headache and other facial pain were not considered for analysis. Localisation of any pain and severity on a Visual Analogue Scale (VAS) were recorded. Medical conditions associated with or predisposing to painful symptoms (including diabetes, osteoporosis, rheumatic disease, degenerative joint disease, arthritis and disk herniation) were checked by examination and clinical records of patients. Finally, the Beck Depression Inventory was administered to assess depression, a variable with a potentially confounding effect on pain. ${ }^{14} 15$

Single and multiple explanatory variable logistic regression models with pain ( 1 , present; 0 , absent) as the outcome

Abbreviations: UPDRS, Unified Parkinson's Disease Rating Scale; VAS, Visual Analogue Scale
Parkinson's disease was diagnosed according to published criteria. ${ }^{13}$ Patients with cognitive impairment (Mini Mental 


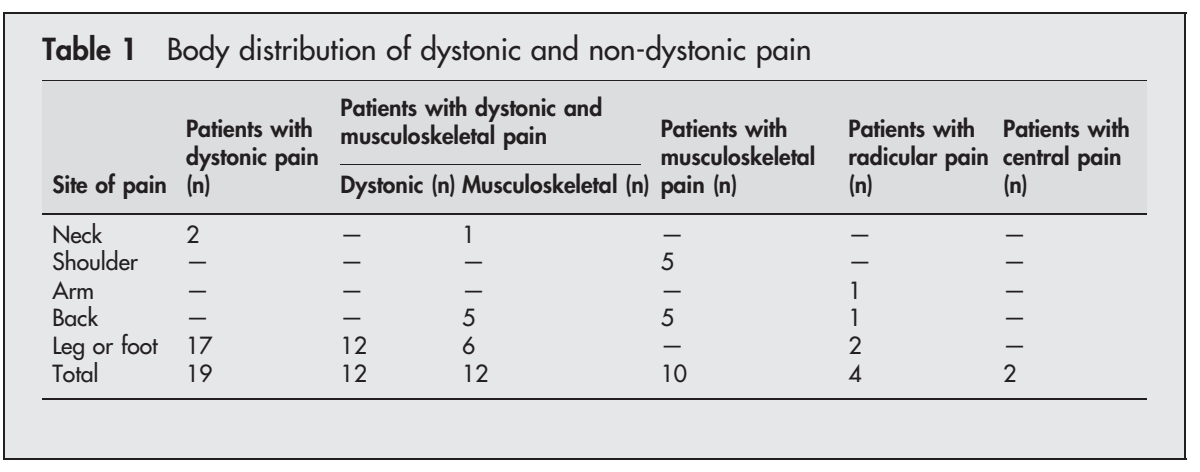

variable were used to check the association with levodoparelated motor complications and the possible confounding effect of relevant demographic and clinical variables ${ }^{16}$. Age, duration of disease, UPDRS motor score, duration of levodopa treatment and current levodopa dosage were analysed as continuous variables; male sex, akinetic rigid dominant phenotype, use of dopamine agonists, depression and medical conditions possibly associated with painful symptoms were analysed as single indicator variables ( 1 , present; 0, absent). Odds ratios (ORs), two-sided 95\% confidence intervals (CIs) and p values were calculated with the STATA 8 package, $\mathrm{p}<0.05$ was considered to be significant. Study power was calculated with the Schlesselmann equation for unmatched studies with an unequal casecontrol ratio. ${ }^{17}$

\section{RESULTS}

A total of 117 patients with Parkinson's disease met the eligibility criteria during the study period, all of whom participated in the study. There were 67 women and 50 men with a mean age of 69.4 (SD 8.1) years with mean duration of disease of 6.5 (SD 5.3) years. Parkinsonian signs were bilateral in 59 patients and unilateral in 58 . The akinetic rigid dominant phenotype was observed in 46 patients and the tremor dominant phenotype in 71. All patients were under pharmacological treatment: levodopa alone in 77 patients, dopamine agonists alone in 34, and levodopa and dopamine agonists in 6 . The group mean UPDRS score part III during the on state was 17.3 (SD 12). A total of 45 patients had dyskinesia $(n=16)$, motor fluctuations in parkinsonian disability $(n=12)$ or both $(n=17)$. When compared with those without motor complications, these patients had longer disease duration (9.6 (6.8) $v 4.5$ (2.8) years, $\mathrm{p}<0.0001)$, greater severity of disease on UPDRS part III (20.3 (13.6) $v$ 15.1 (10.8), $\mathrm{p}=0.024)$, longer duration of levodopa treatment (7.2 (7.4) $v 2.9$ (3.1) years, $\mathrm{p}<0.0001)$ and higher levodopa dosage (683 (385) v 372 (363) mg, p<0.0001).

At the time of study, 47 (40\%) patients reported pain from at least 2 months. None of them referred to the development of pain before starting drugs against Parkinson's disease. Painful states could be classified into dystonic $(\mathrm{n}=19)$ and non-dystonic $(n=16)$, with both categories coexisting in 12 patients. Table 1 shows the body distribution of pain. Dystonic pain referred to dystonic body parts, including the foot, leg and neck. Mean pain intensity on a VAS was 7.1 (SD 1.4). Non-dystonic pain was classified as musculoskeletal $(\mathrm{n}=22)$, radicular or neuritic $(\mathrm{n}=4)$, and primary central $(\mathrm{n}=2)$ pain. No patient had akatisia. Musculoskeletal pain affected the legs $(n=6)$, the lumbar region $(n=10)$, the shoulder $(\mathrm{n}=5)$ and the neck $(\mathrm{n}=1$; table 1$)$. Mean pain severity on a VAS was 6.5 (SD 1.5). Radicular or neuritic pain affected the legs $(n=2)$, the lumbar region $(n=1)$ and the $\operatorname{arm}(n=1)$, with a mean VAS severity of 5.3 (SD 3). The two patients with central pain both checked pain intensity as 8 on a VAS.
Of the 58 patients with unilateral and 59 with bilateral Parkinson's disease, pain was reported by 20 and 27 patients, respectively $\left(\chi^{2}\right.$, non-significant). Among the 20 patients with unilateral Parkinson's disease and with pain, 14 reported pain on the same side as that affected by the disease, 2 on the opposite side and 4 mostly bilateral.

Complaints of dystonic pain occurred during maximal parkinsonian disability in 17 patients or were associated with peak dose $(n=11)$ beginning-dose or end-dose $(n=3)$ dystonia. Reduction in dystonia related to changes in levodopa resulted in decreased pain. Even non-dystonic pain, in particular musculoskeletal pain, was commonly experienced when parkinsonian disability was maximal $(n=19)$, or after a dose of levodopa at the beginning or at the end of its effect $(n=9)$. Keeping the patient in the on state reduced, but did not eliminate, complaints of non-dystonic pain in most cases.

Table 2 shows the demographic and clinical features of patients with Parkinson's disease with or without pain. The two groups did not differ for most investigated variables except for duration of Parkinson's disease, frequency of dyskinesia and motor fluctuations, duration of levodopa treatment and levodopa dosage (table 2). Our study had an estimated $>80 \%$ chance of detecting three times modification in the risk of pain with $\alpha=0.05$ (two sided) for other medical conditions and depression.

The logistic regression model that included all the investigated variables yielded a significant association of pain with motor complications (adjusted OR 5.7; 95\% CI 2 to 16.5; $\mathrm{p}=0.001)$ but not with duration of Parkinson's disease (adjusted OR $1.1 ; 95 \%$ CI 0.9 to $1.4 ; \mathrm{p}=0.3$ ), UPDRS part III (adjusted OR 1.1; 95\% CI 0.97 to $1.15 ; \mathrm{p}=0.3$ ), duration of levodopa treatment (adjusted OR 0.9; 95\% CI 0.7 to 1.1; $\mathrm{p}=0.4$ ) and levodopa dosage (adjusted OR 1.0; 95\% CI 0.99 to $1.001 ; \mathrm{p}=0.4)$. The multiple logistic regression model confirmed the lack of association of other diseases (including diabetes, $\mathrm{n}=5$; osteoporosis, $\mathrm{n}=4$; rheumatic disease, $\mathrm{n}=2$; degenerative joint disease, $\mathrm{n}=13$; arthritis, $\mathrm{n}=7$; and disk herniation, $\mathrm{n}=1$ ) and depression ( 11 patients, 5 with and 6 without pain, were under drug treatment for depression) with pain (not shown). When data were stratified by type of motor complications, we obtained a significant association for both motor fluctuations (adjusted OR 8.6; 95\% CI 2.1 to 35.9; $\mathrm{p}=0.003$ ) and dyskinesia (adjusted OR 5.1; 95\% CI 1.6 to $15.7 ; \mathrm{p}=0.005$ ).

Severity of pain, measured on a VAS, and severity of motor complications, as assessed by UPDRS part IV (Spearman's correlation coefficient $0.31, \mathrm{p}<0.05)$, were significantly correlated.

A separate analysis of dystonic and non-dystonic pain yielded results similar to those obtained on considering all pain types. When adjusting for non-dystonic pain (as a proportion of patients with dystonic pain reported nondystonic pain), dystonic pain was significantly associated with motor complications (OR 4.7; 95\% CI 1.9 to 11.9; 
Table 2 Comparison of patients with Parkinson's disease with and without pain

\begin{tabular}{|c|c|c|c|c|}
\hline & $\begin{array}{l}\text { Patients with pain } \\
(n=47)\end{array}$ & $\begin{array}{l}\text { Patients without } \\
\text { pain }(n=70)\end{array}$ & $\begin{array}{l}\text { Odds ratio ( } 95 \% \\
\text { confidence interval) }\end{array}$ & p Value \\
\hline Sex (men/women) & $19 / 28$ & $31 / 39$ & 0.85 (0.4 to 1.8$)$ & 0.7 \\
\hline Age (years) & $69.7(8.7)$ & $69.2(7.6)$ & $1.1(0.96$ to 1.06$)$ & 0.7 \\
\hline Disease duration (years) & $8.2(6.6)$ & $5.3(3.8)$ & 1.1 (1.03 to 1.2$)$ & 0.008 \\
\hline $\begin{array}{l}\text { Akinetic rigid dominant } v \text { tremor } \\
\text { dominant phenotype }\end{array}$ & $17 / 30$ & $29 / 41$ & 0.80 (0.4 to 1.7$)$ & 0.57 \\
\hline UPDRS III during the on state & $19.5(13.5)$ & $15.9(10.8)$ & 1.02 (0.99 to 1.05$)$ & 0.13 \\
\hline UPDRS III tremor score & $1.5(2.6)$ & $1.1(1.5)$ & $1.1(0.89$ to 1.34$)$ & 0.37 \\
\hline UPDRS III rigidity score & $3.3(3.3)$ & $2.4(2.4)$ & $1.05(0.93$ to 1.2$)$ & 0.43 \\
\hline UPDRS III bradykinesia score & $7.8(5.6)$ & $6.9(5)$ & $1.04(0.96$ to 1.1$)$ & 0.33 \\
\hline Motor complications & 28 & 17 & $6.5(2.8$ to 14.8$)$ & $<0.0001$ \\
\hline Motor fluctuations* & 19 & 10 & $6.3(2.4$ to 15.9$)$ & $<0.0001$ \\
\hline Dyskinesia* & 22 & 11 & $7.7(2.3$ to 17.5$)$ & $<0.0001$ \\
\hline Dopamine agonists & 15 & 25 & $0.9(0.4$ to 2.2$)$ & 0.9 \\
\hline Levodopa treatment & $5.9(6.8)$ & $3.6(4.4)$ & $1.1(1.001$ to 1.16$)$ & 0.045 \\
\hline Levodopa dosage (mg) & $657(320)$ & $617(357)$ & $\begin{array}{l}1.001 \text { (1.0004 to } \\
1.02)\end{array}$ & 0.042 \\
\hline Other diseases & 14 & 18 & $1.4(0.66$ to 2.93$)$ & 0.4 \\
\hline Depression & 14 & 19 & $1.1(0.5$ to 2.5$)$ & 0.8 \\
\hline
\end{tabular}

UPDRS, Unified Parkinson's Disease Rating Scale.

Values are mean (SD) unless otherwise stated.

*Patients with pain $(n=13)$ and without pain $(n=4)$ had both motor fluctuations and dyskinesia.

$\mathrm{p}=0.001)$. Likewise, on adjusting for dystonic pain, a significant association of motor complications with both non-dystonic pain overall (OR 4.4; 95\% CI 1.7 to 11.5; $\mathrm{p}=0.003$ ) and musculoskeletal pain alone (OR 2.2; 95\% CI 1.1 to $8.9 ; \mathrm{p}=0.03$ ) was obtained. The significant association of motor complication with different pain types did not change noticeably after controlling for the other investigated demographic and clinical variables (not shown).

\section{DISCUSSION}

The data presented here confirmed that pain represents a significant feature of Parkinson's disease and that pain symptoms can be classified in clinically distinct subtypes that sometimes overlap. When pain was assessed, all patients were taking drugs against Parkinson's disease, and none of them reported pain before the onset of treatment. Although the retrospective assessment may have biased the observation, this finding is in agreement with the rarity of pain in drug-naive patients with Parkinson's disease, as highlighted by previous studies. ${ }^{15}$ Our analysis also indicated that the association between pain and motor complications (including both motor fluctuations and dyskinesia) was independent of several demographic and clinical variables related to Parkinson's disease, medical diseases potentially associated with painful states and depression. The apparent relationship between pain and duration of disease, duration of levodopa treatment and levodopa dosage resulted from confounding. The lack of association between pain and medical conditions associated with pain in the general population indirectly supports pain as a representative feature of Parkinson's disease. It must be emphasised that the frequency of certain types of painnamely, musculoskeletal or rheumatic pain- is also considerable in the general population, and whether it is more frequently encountered in people with Parkinson's disease than in age-matched controls needs to be fully evaluated. Comparing patients with Parkinson's disease and healthy controls would help to better delineate the cause of non-dystonic pain and its relationships with other symptoms of Parkinson's disease.

The positive association between pain and motor complications may have several explanations. Theoretically, pain may reflect more severe disease associated with motor complications. But we failed to find any marked association between pain and severity of Parkinson's disease as measured by UPDRS part III. Pain possibly is, at least partly, the consequence of the frequent or prolonged muscle hyperactivity associated with dyskinesia and motor fluctuations. Although we found a noticeable correlation between the severity of pain and that of motor complications, the observation that a proportion of patients with unilateral Parkinson's disease reported pain even, or exclusively, on the unaffected side indicates that pain cannot be considered merely a consequence of motor complications. Alternatively, pain and motor complications may share pathophysiological mechanisms. The lack of association between pain and risk factors for motor complications, implicating a disturbance of dopaminergic transmission such as duration of levodopa treatment and levodopa dose, raises the possibility that factors beyond dopaminergic transmission contribute to the pain phenomena in patients with Parkinson's disease. Supporting this view, it was recently observed that the heat pain threshold is considerably lowered in patients with Parkinson's disease with or without pain, regardless of whether they are in an off or on state. ${ }^{18}$ Nondopaminergic basal ganglia neurotransmitter systems (such as $\gamma$ aminobutyric acid, glutamate and enkephalins), possibly implicated in the pathogenesis of motor complications, may also contribute to pain. ${ }^{19-21}$

Despite pain in Parkinson's disease being classified into distinct syndromes, dystonic and non-dystonic pain overlapped in a proportion of patients and both were associated with motor complications. These findings and the recent observation that patients having Parkinson's disease with and without pain may have low heat pain threshold suggest that patients with Parkinson's disease may be predisposed to developing pain. ${ }^{18}$

If so, locoregional factors may determine, at least partly, the heterogeneity of the state of pain. These may be dystonic muscle spasms and postural abnormalities secondary to rigidity and bradykinesia. In our sample, in fact, dystonic pain always referred to dystonic body parts, but for most patients their musculoskeletal pain referred to body parts in which rigidity or bradykinesia is usually more marked (the lumbar region and the shoulder) and which produces trunk postural abnormalities (camptocormia). ${ }^{22}{ }^{23}$

Although this was not a population-based study, we corrected for a bias in case selection by recruiting all consecutive patients with Parkinson's disease who met the eligibility criteria during the study period. Our case population had clinical and demographic features similar to other clinical and population-based series, and was probably representative of Parkinson's disease. As a service-based 
study, our survey probably overestimated the frequency of pain. The overall frequency of patients with pain and the distribution of pain subtypes were, however, comparable to those in previous series. ${ }^{1578}$ Another possible concern could arise from the clinical heterogeneity of complaints of pain and variety of motor complications. We assessed dyskinesia with UPDRS part IV, which does not discriminate between monophasic and diphasic dyskinesia. This point deserves further ad hoc investigation. Moreover, we could not analyse in detail the relationship between pain subtypes and different motor complications owing to lack of statistical power. A larger sample size and a multicentre approach would be required to consider this issue. Nevertheless, we checked the most frequent pain types (namely, dystonic and musculoskeletal pain) for their relationship with motor complications by using logistic regression models to avoid confounding by relevant demographic and clinical variables.

Despite the foregoing limitations, our findings counsel for pain as an important feature of Parkinson's disease in association with motor complications. The results of this study may have implications for understanding pain mechanisms in Parkinson's disease, with the ultimate aim of designing strategies to prevent or cure pain in patients with Parkinson's disease.

\section{Authors' affiliations}

M Tinazzi, C Del Vesco, N Smania, A Fiaschi, Dipartimento di Scienze Neurologiche e della Visione, Sezione di Neurologia Riabilitativa, Università di Verona, Verona, Italy S Ottaviani, G Moretto, Unita Operativa Neurologia Ospedale Civile Borgo Trento, Verona

E Fincati, Clinica Neurologica Policlinico GB Rossi, Verona

D Martino, G Defazio, Dipartimento di Scienze Neurologiche e

Psichiatriche, Università di Bari, Bari, Italy

Competing interests: None declared.

MT and CDV have contributed equally to this work.

\section{REFERENCES}

1 Snider SR, Fahn S, Isgreen WP, et al. Primary sensory symptoms in Parkinsonism. Neurology 1976;26:423-9.

2 Ilson J, Fahn S, Cote L. Painful dystonic spasms in Parkinson's disease. Adv Neurol 1984;40:395-9.

3 Schott GD. Pain in Parkinson's disease. Pain 1985:22:407-11.

4 Quinn NP, Koller WC, Lang AE, et al. Painful Parkinson's disease. Lancet 1986;1:1366-9.

5 Goetz CG, Tanner CaM, Levy M, et al. Pain in Parkinson's disease. Mov Disord 1986;1:45-9.

6 Koller WC. Sensory symptoms in Parkinson's disease. Neurology 1984;34:957-9.

7 Giuffrida R, Vingerhoets FJG, Bogousslavsky J, et al. Syndromes doulourex de la maladie de Parkinson. Rev Neurol (Paris) 2005;161:407-18.

8 Ford B. Pain in Parkinson's disease. Clin Neurosci 1998;5:63-72.

9 Sage JI. Pain in Parkinson's disease. Curr Treat Options Neurol 2004;6:191-200.

10 Chudler EH, Dong WK. The role of the basal ganglia in nociception and pain. Pain 1995;60:3-38.

11 Battista AF, Wolff BB. Levodopa and induced-pain response. A study on patients with parkinsonian and pain syndromes. Arch Intern Med 1973;132:70-4.

12 Urakami K, Takahashi K, Matsushima E, et al. The threshold of pain and neurotransmitter's change on pain in Parkinson's disease. Jpn J Psychiatry Neurol 1990;44:589-93.

13 Gelb DJ, Oliver E, Gilman S. Diagnostic criteria for Parkinson disease. Arch Neurol 1999;56:33-9.

14 Starkstein SE, Preziosi TJ, Robinson RG. Sleep disorders, pain and depression in Parkinson's disease. Eur Neurol 1991;31:352-5.

15 Stein WM, Read S. Chronic pain in the setting of Parkinson's disease and depression. J Pain Symptom Manage 1997;14:255-8.

16 Hosmer DW, Lemeshaw S. Applied logistic regression. New York: Wiley, 1989.

17 Schlesselman JJ. Valid selection of subjects in case-control studies. J Chronic Dis 1985; 38:549-50.

18 Dialdetti R, Shifrin A, Rogowski Z, et al. Quantitative measurement of pain sensation in patients with Parkinson disease. Neurology 2004;62:2171-5.

19 Hallett PJ, Standaert DG. Rationale for use of NMDA receptor antagonists in Parkinson's disease. Pharmacol Ther 2004;102:155-74.

20 Henry B, Duty S, Fox SH, et al. Increased striatal pre-proenkephalin B expression is associated with dyskinesia in Parkinson's disease. Exp Neurol 2003; 183:458-68

21 Brotchie JM. Non dopaminergic mechanisms in levodopa-induced dyskinesia. Mov Disord 2005;20:919-31.

22 Holler I, Dirnberger G, Pirker W, et al. Camptocormia in idiopathic Parkinson's disease. (123)I beta-CIT SPECT and clinical characteristics. Eur Neurol 2003;50:118-20.

23 Fahn S. Description of Parkinson's disease as a clinical syndrome. Ann N Y Acad Sci 2003;991:1-14. 